\title{
Interrelations entre les streptocoques du groupe B qui provoquent des infections chez l'homme et les bovins $\left.{ }^{*}\right)$
}

\author{
par \\ G. HAHN, W. HEESCHEN, J. REICHMUTH \\ et A. TOLLE \\ Institut für Hygiene des Bundesanstalt für Milchforschung in Kiel \\ Directeur : Prof. Dr A. TOLLE
}

Les risques que comporte la consommation du lait cru en nature, sans chauffage préalable destiné à détruire les germes pathogènes éventuellement présents, ont fait l'objet, en France comme dans d'autres pays, de nombreuses discussions et controverses qui se sont déroulées dans diverses enceintes; hygiénistes et professionnels laitiers ont échangé leurs arguments dans un sens ou dans l'autre.

Il nous a semblé que le travail des chercheurs allemands, qui après un rappel de faits connus apporte des observations originales et intéressantes, méritait d'être versé au dossier.

Nous sommes reconnaissants au professeur Tolle et à ses collègues d'avoir bien voulu nous autoriser à publier ici la traduction de leur article et nous espérons que ce travail, qui met en lumière les risques indéniables de la consommation du lait cru, retiendra l'attention des lecteurs de la Revue Le Lait.

M. Plommet et G. Mocouot.

\section{INTRODUCTION}

Streptococcus agalactiae est, aujourd'hui encore, l'un des agents pathogènes les plus fréquemment mis en évidence dans les cas de mammite chez les bovins. Cette espèce microbienne est également isolée dans des cas de maladies graves chez l'homme et le nombre de ces cas va en croissant. Les relations épidémiologiques suscepti-

(*) 9. Kongress der Deutschen Veterinärmedizinischen Gesellschaft, Bad Nauheim 25-27 März 1971, Fortschritte der Veterinärmedezin, 17, 9, 189-197, Paul Parey Berlin und Hamburg, 1972. 
bles d'exister entre les infections par les streptocoques du groupe B chez l'homme et chez les bovins ont été depuis longtemps soupçonnées mais on n'a pas encore pu les mettre en évidence d'une façon décisive.

Plummer (1935), Erbslöh et Grün (1949), Seelemann (1963) et Spasic (1966) ont déjà publié des travaux sur les infections chez l'homme, vraisemblablement provoquées par des bovins ou par la consommation de lait cru. D'autres publications de Møller (1960), Livoni et Molgaard (1965) concernent les infections des bovins résultant d'un contact avec des porteurs de germes humains.

\section{POSITION DU PROBLEME}

Pour élucider les interactions épidémiologiques entre les infections par les streptocoques du groupe B chez les bovins et chez l'homme, nous avons réalisé une série d'études expérimentales en vue d'apporter une réponse aux questions suivantes :

1) Peut-on mettre en évidence des streptocoques du groupe B dans le lait de consommation pasteurisé et cru ?

2) Existe-t-il ou non des différences entre les caractères biologiques des souches isolées chez l'homme et chez les bovins ?

3) Peut-on provoquer une infection expérimentale chez les bovins avec des souches " humaines "?

4) Existe-t-il ou non une relation entre les infections par les streptocoques du groupe B observées chez l'homme et la consommation du lait cru par les patients souffrant de telles infections ?

Nous avons pris comme hypothèse de travail que la population humaine peut être contaminée par le lait cru, que l'agent pathogène s'adapte aux conditions biochimiques de milieu qui existent chez le nouvel hôte (par exemple l'agent pathogène perd la faculté de fermenter le lactose) et qu'à l'intérieur d'une population humaine, le dit agent se transmet horizontalement et verticalement.

\section{PRESENCE DE STREPTOCOQUES DU GROUPE B CHEZ L'HOMME ET LES BOVINS}

\section{Chez les bovins}

Par rapport au nombre total d'échantillons étudiés, la proportion des échantillons positifs pour Streptococcus agalactiae varie de 0,4 à 6,9 p. 100. Par rapport au nombre total de streptocoques responsables de mammites et isolés de la mamelle, la proportion est de 23,1 à 84,8 p. 100 (Shimzu, 1959 ; Spasic, 1966 ; Møller, 1967 ; 
Jelinkova et Heeschen, 1969 ; Institut Fédéral de la Recherche Laitière, Kiel, 1969).

Au cours de la période s'étendant de 1965 à 1970, le laboratoire central de recherches sur les streptocoques de Kiel a classé 11742 souches isolées des glandes mammaires de bovins. Parmi ces souches, 1921 (16,9 p.100) pouvaient être classées dans le groupe sérologique $B$.

\section{Chez l'homme}

On a trouvé des streptocoques du groupe B chez des sujets sains et chez des malades. D'après les données de la littérature mondiale la proportion de streptocoques du groupe B par rapport au nombre total de streptocoques isolés va de 0,2 à 25,2 p. 100 (Müller, 1967).

L'isolement des streptocoques du groupe $\mathrm{B}$ à partir des régions urogénitales est de loin le plus important. Ces mêmes streptocoques du groupe B ont été également mis en évidence dans la région buccale, dans la gorge et dans l'appareil respiratoire supérieur. Plus rarement on les a isolés du sang, du système nerveux central, de la glande mammaire et d'autres organes (Livoni, 1965).

L'importance clinique de ces streptocoques peut se résumer ainsi : on trouve essentiellement Streptococcus agalactiae dans les cas d'infections puerpérales et dans le cas d'autres maladies de la région urogénitale chez la femme (Hood, 1961 ; Eickhoff, 1964 ; Kexel, 1965). On comprend qu'il existe ainsi une transmission au nouveau-né : c'est ce que Hood (1961), Kexel et Beck (1965) et Schröter (1968) ont pu mettre en évidence. Les maladies typiques du nourrisson sont les cas de septicémie et de méningite à évolution foudroyante, la plupart du temps mortelles (Wahl et al., 1962 ; Kexel et Schönbohm, 1965 ; Turner, 1965 ; Gluck et al., 1966 ; Winterbauer, 1966 ; Canet et al., 1967 ; Albrecht et al., 1969). Eickhoff (1964) a pu mettre en évidence, au cours d'observations cliniques poursuivies pendant 2 années, que les streptocoques du groupe B étaient les agents responsables de 25 p. 100 des cas de septicémie chez les nourrissons. D'après le nombre de cas observés les infections de la gorge et les inflammations purulentes nonspécifiques d'autres organes présentaient une importance moindre.

\section{NOS ETUDES PERSONNELLES}

\section{Matériel et méthodes}

1) Recherche de Streptococcus agalactiae dans le lait de consommation pasteurisé et cru. 
Dans le cadre d'études représentatives menées dans l'ensemble de l'Allemagne Fédérale, on a étudié 271 échantillons de lait de consommation pasteurisé et 3465 échantillons de lait de consommation cru, provenant des fournitures de lait cru patenté.

La conservation des échantillons de lait en vue de l'examen bactériologique a été obtenue grâce à l'addition d'un lyophilisat de sorbate de potassium, d'acide borique, de glycérine et de bleu de méthylène (Heeschen et al., 1969). On a incubé $0,5 \mathrm{ml}$ de l'échantillon ainsi conservé dans $5 \mathrm{ml}$ d'un bouillon contenant du pourpre de bromocrésol, du streptosel et de la kanamycine, pendant 24 h à $37^{\circ} \mathrm{C}$ (Heeschen et al., 1968). Lors du virage de l'indicateur coloré du bleu-violet au jaune, on a étalé, à l'aide d'une anse, la culture sur une gélose au sang et on a incubé à nouveau pendant $24 \mathrm{~h}$ à $37^{\circ} \mathrm{C}$.

\section{TABLEAU 1}

Classification de 1078 souches humaines de streptocoques du groupe B selon leur provenance

(données rassemblées par le laboratoire central de recherches sur les streptocoques de Kiel)

\begin{tabular}{l|r|r}
\hline - Région urogénitale & 754 & 69,9 p. 100 \\
- Appareil respiratoire supérieur & 145 & 13,4 p. 100 \\
- Appareil circulatoire & 21 & 1,9 p. 100 \\
- Système nerveux central & 15 & 1,4 p. 100 \\
- Glande mammaire & 5 & 0,5 p. 100 \\
- Cas divers & 138 & 12,8 p. 100 \\
\hline Total & 1078 & 100,0 p. 100 \\
\hline
\end{tabular}

En même temps, on a également déposé $0,1 \mathrm{ml}$ du même échantillon de lait directement sur une gélose TKT que l'on a incubée pendant $24 \mathrm{~h}$ à $37^{\circ} \mathrm{C}$.

Pour le diagnostic de la présence de Streptococcus agalactiae, on a pris comme critère décisif le résultat de la réaction sérologique de précipitation.

2) Etude des caractères biologiques.

Cent-une souches isolées sur des patients humains provenant de 7 cliniques et villes et 102 souches isolées chez les bovins provenant de 
7 troupeaux de l'Allemagne du nord, ont été classées d'un point de vue biochimique et sérologique (Heeschen et al., 1968 ; Hahn et al., 1970 ; Jelinkova et Heeschen, 1969).

Le caractère pathogène pour la souris a été testé au moyen d'une culture en bouillon incubée pendant $24 \mathrm{~h}$ à $37^{\circ} \mathrm{C}$. On a utilisé des taux de dilution de cette culture allant jusqu'au 1/50. Pour chaque taux de dilution, on a utilisé 3 souris qui ont reçu $0,5 \mathrm{ml}$ de la culture diluée par injection intra-péritonéale. On a gardé les animaux en observation pendant 7 jours. On a réalisé une culture à partir du sang des souris mortes ou sacrifiées par la suite.

L'isolement et l'identification des acides aminés de la paroi cellulaire ont été faits selon la technique indiquée par Colman et Williams (1965).

\section{3) Infection expérimentale chez les bovins.}

On a contaminé 12 vaches en lactation avec une souche du type sérologique Ia. La souche incubée en bouillon de culture pendant $24 \mathrm{~h}$ à $37^{\circ} \mathrm{C}$ a été mise en suspension dans le lait même des vaches. Chaque vache a reçu une dose contaminante allant de 1940 à 712000 germes, administrée en infusion intramammaire. Avant et après la contamination, on a déterminé le nombre de cellules somatiques et le comportement général des animaux et on a contrôlé la quantité de lait sécrétée. La durée de l'expérience a été de 3 semaines.

4) Etudes sur les infections par les streptocoques du groupe B chez l'homme en relation avec la consommation du lait cru.

L'hypothèse selon laquelle il s'agit, dans le cas des streptocoques du groupe B isolés chez l'homme et chez les bovins, d'une même espèce pathogène vis-à-vis des humains comme vis-à-vis des bovins devait être confirmée par une enquête et à l'aide d'un matériel très important que possédait le laboratoire central de recherches sur les streptocoques de Kiel. On a déterminé l'origine de 250 souches de streptocoques du groupe B isolées chez l'homme et on a effectué les recherches nécessaires dans les archives de 5 hôpitaux de Hambourg, Krefeld et Mannheim pour se procurer les noms et les adresses des patients correspondants.

Un questionnaire a été envoyé à ces patients, questionnaire dont les 6 premières questions avaient pour but de savoir si le patient avait eu éventuellement des contacts directs ou indirects avec des bovins (à son domicile, dans sa profession ou en d'autres occasions). Les 2 questions suivantes avaient pour but de déterminer s'il existait une possibilité de transmission des streptocoques du groupe B par l'intermédiaire du lait cru ou du lait et des produits laitiers en général, 137 des 250 personnes interrogées ont répondu à ce questionnaire et on a donc pu exploiter leurs réponses. 


\section{RESULTATS}

1) Les streptocoques du groupe B dans le lait de consommation. Aucun streptocoque du groupe B n'a pu être mis en évidence dans les échantillons de lait pasteurisé étudiés.

Par contre, en ce qui concerne les échantillons provenant des troupeaux produisant du lait cru patenté, conditionné en emballage pour la vente et mis en vente comme lait cru patenté, on a trouvé des streptocoques du groupe B dans 20,4 p. 100 des échantillons en moyenne. Le tableau 2 montre la répartition des résultats pour les différentes sortes d'échantillons.

TABLEAU 2

Présence de Streptococcus agalactiae dans 3465 échantillons de lait cru (lait cru patenté)

\begin{tabular}{l|r|r|c}
\hline & \multicolumn{2}{|c|}{ Nombre } & \\
\cline { 2 - 3 } & échantillons & $\begin{array}{c}\text { strepto- } \\
\text { coques B }\end{array}$ & \\
\cline { 2 - 3 } & & & \\
- Lait de quartiers séparés & 20 & 3 & 15,0 \\
- Lait d'une vache & 2801 & 589 & 21,0 \\
- Lait de mélange & 215 & 50 & 23,2 \\
- Lait de consommation & 363 & 54 & 14,9 \\
- Cas divers & 66 & 10 & 15,1 \\
\hline Total & 3465 & 706 & 20,4 \\
\hline
\end{tabular}

Dans ce contexte, on a comparé l'isolement des streptocoques du groupe B par étalement direct sur une gélose TKT et par enrichissement en milieu liquide (bouillon Streptosel).

Pour 372 échantillons de lait, on a pu isoler 150 fois des streptocoques du groupe B par enrichissement en milieu liquide (40,3 p. 100) et seulement 28 fois ( 7,5 p. 100) par étalement sur gélose TKT. 
TABLEAU 3

Spectre de propriétés biochimiques différenciant les souches humaines des souches bovines des streptocoques du groupe B

\begin{tabular}{|c|c|c|c|c|c|c|c|}
\hline \multirow{3}{*}{ Critères } & \multicolumn{3}{|c|}{ Souches humaines } & \multicolumn{3}{|c|}{ Souches bovines } & \multirow{3}{*}{$\begin{array}{l}\text { Numéros des } \\
\text { références } \\
\text { bibliogra- } \\
\text { phiques } \\
\text { correspon- } \\
\text { dantes }\end{array}$} \\
\hline & \multicolumn{2}{|c|}{ Nombre } & \multirow{2}{*}{$\begin{array}{c}\text { Positifs } \\
\text { p. } 100\end{array}$} & \multicolumn{2}{|c|}{ Nombre } & \multirow{2}{*}{$\begin{array}{c}\text { Positifs } \\
\text { p. } 100\end{array}$} & \\
\hline & Total & Positifs & & Total & Positifs & & \\
\hline - Fermentation du lactose & $\begin{array}{r}31 \\
110 \\
20 \\
109\end{array}$ & $\begin{array}{l}11 \\
28 \\
12 \\
32\end{array}$ & $\begin{array}{l}35,5 \\
25,4 \\
60,0 \\
29,3\end{array}$ & $\begin{array}{r}38 \\
34 \\
50 \\
102\end{array}$ & $\begin{array}{r}24 \\
34 \\
50 \\
100\end{array}$ & $\begin{array}{r}64,8 \\
100,0 \\
100,0 \\
98,0\end{array}$ & $\begin{array}{r}6 \\
27 \\
25 \\
*\end{array}$ \\
\hline - Fermentation de la glycérine & $\begin{array}{r}31 \\
20 \\
109\end{array}$ & $\begin{array}{l}24 \\
12 \\
55\end{array}$ & $\begin{array}{l}77,4 \\
60,0 \\
50,4\end{array}$ & $\begin{array}{r}38 \\
50 \\
102\end{array}$ & $\begin{array}{r}13 \\
9 \\
65\end{array}$ & $\begin{array}{l}34,2 \\
18,0 \\
63,7\end{array}$ & $\begin{array}{r}6 \\
25 \\
*\end{array}$ \\
\hline $\begin{array}{l}\text { - Croissance dans le lait bleu de } \\
\text { méthylène } \\
\text { 1:20 } 000\end{array}$ & $\begin{array}{r}31 \\
109\end{array}$ & $\begin{array}{r}3 \\
67\end{array}$ & $\begin{array}{r}9,7 \\
61,4\end{array}$ & $\begin{array}{r}38 \\
102\end{array}$ & $\begin{array}{l}16 \\
32\end{array}$ & $\begin{array}{l}42,1 \\
31,3\end{array}$ & $\begin{array}{c}6 \\
*\end{array}$ \\
\hline 1: 5000 & 31 & 17 & 55,1 & 38 & 34 & 89,5 & 6 \\
\hline - Thermorésistance $60^{\circ} \mathrm{C}$ & 118 & 40 & 33,9 & 104 & 17 & 16,3 & * \\
\hline
\end{tabular}

* Résultats obtenus par les auteurs du présent article. 


\section{TABLEAU 4}

Etudes des différences dans la répartition des types d'antigènes entre souches humaines et souches bovines de streptocoques du groupe B

\begin{tabular}{|c|c|c|c|c|c|c|c|}
\hline \multirow{3}{*}{ Critères } & \multicolumn{3}{|c|}{ Souches humaines } & \multicolumn{3}{|c|}{ Souches bovines } & \multirow{3}{*}{$\begin{array}{l}\text { Numéros des } \\
\text { références } \\
\text { bibliogra- } \\
\text { phiques } \\
\text { correspon- } \\
\text { dantes }\end{array}$} \\
\hline & \multicolumn{2}{|c|}{ Nombre } & \multirow{2}{*}{$\begin{array}{c}\text { Positifs } \\
\text { p. } 100\end{array}$} & \multicolumn{2}{|c|}{ Nombre } & \multirow{2}{*}{$\begin{array}{c}\text { Positifs } \\
\text { p. } 100\end{array}$} & \\
\hline & Total & Positifs & & Total & Positifs & & \\
\hline \multicolumn{8}{|l|}{ Type d'antigène : } \\
\hline - polysaccharidiques & $\begin{array}{l}147 \\
101\end{array}$ & $\begin{array}{r}125 \\
72\end{array}$ & $\begin{array}{c}70-100 \\
85,0 \\
71,3\end{array}$ & $\begin{array}{l}600 \\
102\end{array}$ & $\begin{array}{r}141 \\
13\end{array}$ & $\begin{array}{c}19-50 \\
23,5 \\
12,7\end{array}$ & $\begin{array}{l}21 \\
22 \\
*\end{array}$ \\
\hline - protéiques & $\begin{array}{l}147 \\
101\end{array}$ & $\begin{array}{r}4 \\
12\end{array}$ & $\begin{array}{r}17^{* *} \\
2,8 \\
11,9\end{array}$ & $\begin{array}{l}600 \\
102\end{array}$ & $\begin{array}{r}347 \\
59\end{array}$ & $\begin{array}{c}22-45 \\
57,8 \\
57,8\end{array}$ & $\begin{array}{c}21 \\
22 \\
*\end{array}$ \\
\hline
\end{tabular}

* Résultats obtenus par les auteurs du présent article.

** Différentes valeurs ont été observées, jusqu'à 17 p. 100. 
2) Caractères biologiques des streptocoques du groupe B isolés des bovins et de l'homme.

Les caractères biochimiques pour lesquels on peut établir une distinction significative ainsi que les résultats de la sérotypie observés pour les souches d'origine humaine et pour les souches d'origine bovine sont donnés dans les tableaux suivants.

Le tableau 3 montre que les souches humaines et bovines se distinguent significativement par leur aptitude à fermenter le lactose et la glycérine, par leur résistance vis-à-vis de concentrations croissantes en bleu de méthylène et par leur thermorésistance.

Les résultats de la sérotypie montrent que les antigènes polysaccharidiques Ia-III (selon Lancefield) prédominent chez les souches humaines, alors que chez les souches bovines les antigènes protéiques $\mathrm{R}$ et $\mathrm{X}$ sont mis en évidence beaucoup plus fréquemment (voir tabl. 4).

En ce qui concerne le pouvoir pathogène, les souches humaines sont, de façon régulière, plus pathogènes pour la souris que les souches bovines (tabl. 5).

\section{TABLEAU 5}

Différences observées entre les souches humaines et bovines des streptocoques du groupe B au point de vue de leur pathogénicité pour la souris

\begin{tabular}{|c|c|c|c|c|c|c|}
\hline \multicolumn{3}{|c|}{ Souches humaines } & \multicolumn{3}{|c|}{ Souches bovines } & \multirow{3}{*}{ 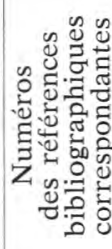 } \\
\hline & & \multirow{2}{*}{ p. 100} & \multicolumn{2}{|c|}{ Nombre } & \multirow{2}{*}{ p. 100} & \\
\hline Total & Positifs & & Total & Positifs & & \\
\hline 20 & 13 & 65,0 & 50 & 2 & 4,0 & 25 \\
\hline 144 & 69 & 48,6 & 283 & 32 & 11,3 & 22 \\
\hline 101 & 90 & 89,8 & 102 & 41 & 40,2 & * \\
\hline
\end{tabular}

* Résultats observés par les auteurs du présent article. 


\section{TABLEAU 6}

Rapports molaires d'amino-acides les plus importants des parois cellulaires cie streptocoques du groupe sérologique B (B : souches bovines, $\mathrm{H}$ : souches humaines)

\begin{tabular}{|c|c|c|c|c|c|c|c|c|c|c|}
\hline Type & Spécificité & Asparagine & Méthionine & Thréonine & Sérine & Muramine & Glutamine & Glycocolle & Alanine & Lysine \\
\hline $1 \mathrm{~b}$ & $\begin{array}{l}\mathrm{B} \\
\mathrm{H}\end{array}$ & $\overline{0,06}$ & $\begin{array}{l}0,24 \\
0,08\end{array}$ & $\begin{array}{l}0,18 \\
0,12\end{array}$ & $\begin{array}{l}0,34 \\
0,39\end{array}$ & $\begin{array}{l}0,43 \\
0,47\end{array}$ & $\begin{array}{l}1,00 \\
1,00\end{array}$ & $\begin{array}{l}0,16 \\
0,10\end{array}$ & $\begin{array}{l}3,05 \\
3,93\end{array}$ & $\begin{array}{l}0,59 \\
1,27\end{array}$ \\
\hline II & $\begin{array}{l}\mathrm{B} \\
\mathrm{H}\end{array}$ & $\begin{array}{l}0,02 \\
0,07\end{array}$ & $\begin{array}{l}0,10 \\
0,14\end{array}$ & $\begin{array}{l}0,07 \\
0,14\end{array}$ & $\begin{array}{l}0,23 \\
0,32\end{array}$ & $\begin{array}{l}0,39 \\
0,37\end{array}$ & $\begin{array}{l}1,00 \\
1,00\end{array}$ & $\begin{array}{l}0,07 \\
0,12\end{array}$ & $\begin{array}{l}3,00 \\
3,07\end{array}$ & $\begin{array}{l}0,92 \\
1,00\end{array}$ \\
\hline $\begin{array}{l}\text { III } \\
\text { (R) }\end{array}$ & $\begin{array}{l}\text { B } \\
\text { H }\end{array}$ & $\begin{array}{l}0,04 \\
0,05\end{array}$ & $\begin{array}{l}0,09 \\
0,17\end{array}$ & $\begin{array}{l}0,09 \\
0,17\end{array}$ & $\begin{array}{l}0,40 \\
0,32\end{array}$ & $\begin{array}{l}0,47 \\
0,47\end{array}$ & $\begin{array}{l}1,00 \\
1,00\end{array}$ & $\begin{array}{l}0,04 \\
0,14\end{array}$ & $\begin{array}{l}3,49 \\
3,22\end{array}$ & $\begin{array}{l}1,05 \\
1,08\end{array}$ \\
\hline (R) & $\begin{array}{l}\mathrm{B} \\
\mathrm{H}\end{array}$ & $\begin{array}{l}0,35 \\
0,03\end{array}$ & $\begin{array}{l}0,13 \\
0,07\end{array}$ & $\begin{array}{l}0,12 \\
0,09\end{array}$ & $\begin{array}{l}0,51 \\
0,29\end{array}$ & $\begin{array}{l}0,44 \\
0,39\end{array}$ & $\begin{array}{l}1,00 \\
1,00\end{array}$ & $\begin{array}{l}0,10 \\
0,05\end{array}$ & $\begin{array}{l}3,29 \\
3,38\end{array}$ & $\begin{array}{l}1,06 \\
1,18\end{array}$ \\
\hline
\end{tabular}


On a constaté des différences également en ce qui concerne la teneur en acides aminés des parois cellulaires isolées. Dans le tableau 6 , les chiffres en caractères gras montrent les rapports molaires (calculés pour 1 molécule d'acide glutamique) présentant entre eux une différence significative.

3) Infection expérimentale des bovins.

En inoculant les 12 vaches en lactation, on a pu provoquer régulièrement des mammites avec un streptocoque du groupe B isolé chez l'homme. $18 \mathrm{~h}$ après l'inoculation, le nombre de leucocytes par $\mathrm{ml}$ de lait atteignait des valeurs allant de 2,6 à 30 millions (tabl. 7). Les animaux présentaient en outre régulièrement une inflammation aiguë avec fièvre et arrêt immédiat de la sécrétion du lait.

\section{TABLEAU 7}

Augmentation du nombre des cellules du lait (déterminé électroniquement) après l'inoculation à plusieurs vaches laitières d'une dose variable (selon la vache) de streptocoques du groupe B d'origine humaine

( $\mathrm{xg}$ moyenne géométrique)

\begin{tabular}{|c|c|c|c|c|}
\hline \multirow{2}{*}{$\begin{array}{l}\mathrm{N}^{\circ} \\
\text { vache }\end{array}$} & \multirow{2}{*}{$\begin{array}{l}\text { Nombre de } \\
\text { germes } \\
\text { inoculés }\end{array}$} & \multirow{2}{*}{$\begin{array}{c}\text { Nombre de cellules par ml } \\
\text { (en milliers) } \\
\text { xg } 7 \text { jours avant } \\
\text { l'inoculation }\end{array}$} & \multicolumn{2}{|c|}{$\begin{array}{c}\text { Nombre de cellules par ml } \\
\text { (en milliers) } \\
\text { après l'inoculation }\end{array}$} \\
\hline & & & $18 \mathrm{~h}$ & $42 \mathrm{~h}$ \\
\hline $\begin{array}{r}79 \\
80 \\
85 \\
87 \\
88 \\
90 \\
91 \\
95 \\
96 \\
97 \\
105 \\
106\end{array}$ & $\begin{array}{r}3800 \\
282000 \\
712000 \\
3200 \\
3200 \\
1940 \\
5600 \\
5600 \\
5600 \\
282000 \\
712000 \\
712000\end{array}$ & $\begin{array}{r}80 \\
53 \\
189 \\
60 \\
58 \\
89 \\
86 \\
90 \\
70 \\
55 \\
67 \\
53\end{array}$ & $\begin{array}{r}16263 \\
15199 \\
2608 \\
23 \quad 355 \\
6115 \\
6812 \\
30000 \\
13425 \\
25105 \\
30000 \\
27655 \\
10930\end{array}$ & $\begin{array}{l}25901 \\
29655 \\
11667 \\
21650 \\
24081 \\
27987 \\
29472 \\
12329 \\
28993 \\
30283 \\
30000 \\
27234\end{array}$ \\
\hline
\end{tabular}

4) Relations entre les infections par les streptocoques du groupe B chez l'homme et la consommation du lait cru.

A partir des réponses au questionnaire, il n'a pas été possible d'obtenir un indice quelconque d'une relation éventuelle entre d'une part la profession et le domicile des patients et d'autre part un contact avec les bovins et leur infection par les streptocoques du 
groupe B. Par contre, parmi les patients contaminés par les streptocoques $\mathrm{du}$ groupe $\mathrm{B}$, le pourcentage des consommateurs de lait cru était très élevé (on entend par lait cru, le lait de vache nonpasteurisé et le lait cru patenté (voir tabl. 8).

\section{TABLEAU 8}

Consommation de lait de vache cru et de lait cru patenté par 137 patients contaminés par des streptocoques du groupe B

\begin{tabular}{l|c|c|c}
\hline & $\mathrm{A}^{*}$ & $\mathrm{~B}^{*}$ & \\
Lait cru ramassé à la ferme & 4 & 7 & 8,0 p. 100 \\
Lait cru patenté & 12 & 15 & 19,7 p. 100 \\
\hline Total & 16 & 22 & 27,7 p. 100 \\
\hline
\end{tabular}

* A : consommation régulière ; * $\mathrm{B}$ : consommation occasionnelle.

Au total, 27,7 p. 100 des patients avaient bu du lait cru avant leur maladie. En outre 27 des 137 porteurs de streptocoques du groupe B (19,7 p. 100) étaient, avant leur maladie, des consommateurs réguliers ou occasionnels de lait cru patenté.

Le calcul qui suit est basé uniquement sur le lait cru patenté en raison du fait que les conditions de consommation peuvent, dans le cas de ce lait cru patenté, être évaluées avec une bonne précision. On a utilisé pour ce calcul les valeurs suivantes :

- la consommation de lait de consommation est en moyenne de $280 \mathrm{ml}$ par personne et par jour en République Fédérale d'Allemagne,

- la production annuelle de lait cru patenté dans ce même pays est de 30 millions de litres,

- si l'on suppose que les consommateurs de lait cru patenté en consomment également $280 \mathrm{ml}$ par personne et par jour, on en déduit qu'environ 0,5 p. 100 seulement des 60 millions d'habitants de la République Fédérale d'Allemagne boivent de ce lait.

De ces chiffres, on déduit que le rapport du nombre des consommateurs de lait cru patenté au reste de la population est de 0,5 pour 99,5 .

Par contre, d'après le sondage effectué chez les patients atteints d'infections par les streptocoques du groupe B, on observe que parmi ces patients la proportion des consommateurs de lait cru patenté est de 19,7 pour 80,3 . D'après ce sondage, le nombre de consommateurs de lait cru patenté contaminés par des streptocoques du groupe B est donc environ 40 fois plus élevé que ce à quoi l'on devrait 
s'attendre si les proportions de buveurs de lait cru patenté étaient les mêmes dans les deux groupes (population totale d'une part et patients contaminés par des streptocoques du groupe B d'autre part).

\section{DISCUSSION}

Dans l'étude que nous avons réalisée, l'hypothèse de travail supposait que les souches de streptocoques appartenant au groupe sérologique B isolées chez l'homme et chez les bovins avaient une origine commune, mais que ces souches pouvaient modifier leur caractère biologique par adaptation à l'hôte du moment. C'est la glande mammaire de la vache qui a été considérée comme le réservoir de ces agents pathogènes. Cette hypothèse de travail a été étayée aussi bien par l'exploitation de la littérature mondiale que par les études épidémiologiques et expérimentales existantes.

1) Alors que Streptococcus agalactiae est détruit avec certitude par le procédé de pasteurisation officiellement autorisé, on peut mettre en évidence dans le lait cru (par exemple dans le lait cru vendu à la ferme ou dans le lait cru patenté) un pourcentage élevé de streptocoques du groupe B. Les résultats cités plus haut permettent de conclure que, malgré les conditions d'hygiène particulièrement bonnes et le contrôle officiel régulier des exploitations produisant du lait cru patenté, les infections de la glande mammaire et par conséquent du lait, par les streptocoques du groupe B, ne peuvent être évitées avec une garantie suffisante.

2) Les études portant sur la biologie des streptocoques du groupe $\mathrm{B}$ aboutissent à la conclusion que ces agents pathogènes sont transmis des bovins aux humains par l'intermédiaire du lait cru et qu'ils se répandent ensuite à l'intérieur de la population humaine horizontalement et verticalement.

Ceci est de mieux en mieux démontré par l'examen des caractéristiques morphologiques, bactériologiques, biochimiques et sérologiques ainsi que par l'examen de la pathogénicité pour la souris des streptocoques du groupe B chaque fois que des souches sont isolées chez l'homme et chez les bovins. Cependant on doit observer qu'il existe un chevauchement important de ces caractéristiques à l'intérieur de chaque espèce et ce phénomène est l'indice d'une adaptation à un nouveau milieu.

3) Les résultats des infections expérimentales des bovins avec des streptocoques du groupe B isolés chez l'homme prouvent que la même souche peut être pathogène pour l'homme et pour les bovins. Une subdivision en variété bovine et variété humaine ne se justifie donc pas sur le plan pratique au point de vue de la pathogénicité.

4) Une enquête menée chez des patients contaminés par des streptocoques du groupe B a permis de constater qu'une proportion extrêmement importante de ces patients avaient consommé avant leur 
maladie, régulièrement ou occasionnellement, du lait cru. Une comparaison statistique a pu apporter des indications montrant que le risque d'infection par les streptocoques du groupe B est beaucoup plus important chez les personnes qui consomment du lait cru.

L'hypothèse selon laquelle les streptocoques du groupe B isolés chez l'homme et chez les bovins constituent le même agent pathogène est rendue vraisemblable du fait de la fréquence comparable des infections à streptocoques du groupe $\mathbf{B}$ chez l'homme et chez les bovins, par les propriétés biologiques partiellement différentes des souches isolées chez l'homme et chez les bovins, par le pouvoir pathogène des souches humaines pour les bovins et par l'indication statistique d'un risque d'infection plus élevé chez l'homme lié à la consommation du lait cru.

En conclusion, il faudrait envisager, en ce qui concerne la médecine humaine, un approfondissement des études ayant trait aux mesures à prendre vis-à-vis des streptocoques du groupe B dans les soins préventifs recommandés aux femmes enceintes et, pour la médecine vétérinaire, il faudrait prévoir un contrôle plus sévère du lait cru commercialisé en vue de la consommation en l'état.

- Günther (de Bersenbrück) a posé la question suivante : Est-on autorisé à livrer le lait fourni par un cheptel contaminé par des streptocoques même si l'on n'a pas réussi à dépister les streptocoques de mammites ?

- Henker (de Heidelberg) a posé cette deuxième question : Nous avons observé dans l'Etat de Bade-Würtemberg, une augmentation nette des mammites, essentiellement dans les troupeaux de Frisonne Pie-Noire. Vous n'avez parlé que de la transmission des streptocoques du groupe $\mathrm{B}$ d'origine humaine à la mamelle de la vache en lactation (expérience sur 12 vaches). Mais ne connaît-on pas également des infections spontanées des bovins par l'homme, par exemple des trayeurs chez lesquels on a trouvé des streptocoques dans la gorge (certaines de ces personnes se crachant dans les mains avant la traite) ?

- Réponse de Hahn (de l'Institut de Kiel) : Dans la bibliographie, on a trouvé un cas de contamination d'un troupeau par la personne qui s'occupait de l'alimentation des animaux (voir Møller).

\section{Ré s u mé}

La présence de Str. agalactiae (streptocoque du groupe B) en tant qu'agent pathogène, chez les bovins et chez l'homme pour lequel ce streptocoque est la cause de certaines maladies graves, 
a fait l'objet d'une étude sur les infections possibles de l'homme résultant de la consommation du lait.

Cette étude a porté sur la présence de l'agent pathogène dans le lait de consommation, sur les caractéristiques biologiques des souches isolées chez l'homme et chez les bovins, sur la nature pathogène des souches "humaines " pour les bovins ainsi que sur les rapports entre les infections de l'homme par les streptocoques du groupe B et la consommation du lait cru. Elles ont abouti aux résultats suivants :

$1^{\circ}$ Les streptocoques du groupe B n'ont pas été dépistés dans le lait pasteurisé, mais ils ont été trouvés dans le lait cru (lait cru patenté) en moyenne dans 20,4 p. 100 des échantillons examinés. L'origine de l'infection observée a été ainsi établie.

$2^{\circ}$ Les souches bovines et les souches humaines se distinguent nettement par certains caractères biologiques ; cependant le chevauchement observé entre les caractères biologiques ne permet pas d'établir une distinction entre différentes espèces ou variants.

3o Des mammites aiguës, accompagnées de fièvre, peuvent être déclenchées d'une façon régulière chez les bovins par les souches isolées de l'homme.

$4^{\circ}$ Une enquête menée parmi 137 patients atteints d'une infection par les streptocoques du groupe B a permis de fournir des indications précises, vérifiées par un calcul statistique, concernant le fait que la consommation du lait cru augmente d'une façon appréciable les risques d'infection par les streptocoques du groupe B.

Il est nécessaire d'assurer un contrôle plus sévère de la distribution du lait cru de consommation.

Reçu pour publication le 20 janvier 1974.

\section{S u m m a ry}

Str. agalactiae (Group B Streptococcus) is pathogenic for both bovine and man. In man the illness caused by this organism can be particularly serious. A possible mode of infection is by the consumption of milk.

Studies were therefore carried out to determine the presence of the pathogen in milk for consumption, on the biological characteristics of the strains isolated from man and bovine, on the pathogenicity of the human strains for the cow, and on the relationships between human infections caused by Group B streptococci and raw milk consumption. These studies have led to the following results : 
1) Group B streptococci were not detected in pasteurized milk, but they were found in 20,4 p. 100 of the raw milk samples examined (licensed raw milk). The origin of the observed infection was thus established.

2) Bovine and human strains could clearly be distinguished by certain biological characteristics. However, the overlapping of these characteristics did not allow a distinction to be made between different species or variants.

3) Acute mastitis accompanied by fever could regularly be initiated in bovine by strains isolated from man.

4) From an investigation of the case histories of 137 patients suffering from infection by Group B streptococci it was possible to demonstrate statistically the appreciably increased risk of infection by Group B streptococci associated with the consumption of raw milk.

It is necessary to ensure much stricter control of the distribution of raw milk destined for consumption.

\section{Bibliographie}

1. Albrecht (J. H.) u. a. (1969). - Tödliche Neugeborenensepsis durch B-Streptokokken. Med. Klinik, 64, 1168.

2. CANet (J.) u. a. (1967). - Infection néo-natale à streptocoque B. Arch. Franç. Péd., 24, 1045-1053.

3. Colman (G.) and Williams (R. E. O.) (1965), - The cell walls of streptococci. J. gen. Microbiol., 41, 375-387.

4. Eickнoff (T. C.) (1964). - Neonatal sepsis and other infections due to group B B-hemolytic streptococci. New. Engl. J. Med., 271, 1221.

5. ERBSLöH (F.) und GRüN (L.) (1949). - Galtstreptokokken der serolog. Gruppe B nach Lancefield als Erreger der Endocarditis lenta. Dtsch. med. Rdsch., 3, 508-512.

6. El Ghoroury (A. A.) (1950). - Comparative Studies of Group B Streptococci of human and bovine origin. I. cultural and biochemical characters. Amer. J. of Publ. Health, 40, 1273.

7. Gluck (L.), Wood (H. F.) and FouseK (M. D.) (1966). - Septicemia of the newborn. Pediatrics clinics N. America, 13, 1130.

8. Hahn (G.) u. a. (1970). - Streptococcus - Eine Studie zur Struktur, Biochemie, Kultur und Klassifizierung. Kieler Milchw. Forsch. Ber., 22, 333-546.

9. HeEschen (W.) u.a. (1967). - Klassifizierung der Gattung Streptococcus. Zbl. Bakt. I. Orig., 205, 250-259.

10. Heeschen (W.) u. a. (1968). - Die selektive Züchtung und Anreicherung koagulasepositiver Staphylok'kken und euterpathogener Streptokokken in flüssigen Nährböden. Arch. Lebensmittelhyg., 19, 184.

11. Heeschen (W.) u. a. (1969). - Die Konservierung von Milchproben zur bakteriologischen, zytologischen und hemmstoffbiologischen Untersuchung. Milchwirtsch. Forschungsber., 24, 729.

12. Hood (M.) Janney (A.) and Dameron (G.) (1961). - $\beta$-Hämolyt. streptococcus group $\mathrm{B}$ associated with problems of the perinatal period. Americ. $K$. Obstet Gynec., 82, 809-818. 
13. Jelinkova (J.) und Heeschen (W.) (1969). - B-Streptokokken bei Mensch und Rind. 1. Mitt. : Ergebnisse der serologischen Typisierung. Zbl. Bakt. I. Orig., $209,312$.

14. KeXel (G.) (1965). - Uber das Vorkommen der B-Streptokokken beim Menschen. Z. Hyg., 151, 336-348.

15. KexEl (G.) und BecK (K. J.) (1965). - Untersuchungen über die Häufigkeit der B-Streptokokken im Wochenbett. Geburtsh. u. Frauenheilk., 25, 1078-1085.

16. KeXel (G.) und Sснӧлвонм (S.) (1965). - Streptococcus agalactiae als Erreger von Säuglingsmenigitiden. Dtsch. med. Wochenschr., 90, 258-261.

17. Bundesanstalt für Milchforschung (1969). - Kieler Milchwirtschaftliche Forschungsberichte, 21, 211-229.

18. Livoni (P.) u. a. (1965). - Group B streptococcus infections in man and cows. I. Mitt, Ugeskr. Laeg., 127, 43, 1353-1362.

19. Livoni (P.) and MolgaARd (A.) (1965). - Groups streptococcus infections in man and cows. II Mitt. Medlemsbl, danske Dyrlaergeforen, 48, 871-880.

20. Möller (Sv.) (1960). - Mastitisprobleme. Medlemsbl. danske Dyrlaergeforen, 796-799.

21. Müiler (G.) (1967). - Die Typisierung der Streptokokken der Gruppe B. a) I. Mitt. : Bedeutung, Antigenstruktur und Typen der B-Streptokokken. Arch. Exp. Vet. Med., 21, 43-54.

22. Müller (G.) (1967). - b) II. Mitt. : Methoden und Ergebnisse der Typisierung von humanen und bovinen Streptokokken der Gruppe B. Arch. Exp. Vet. Med., 21, 55-64.

23. MüLleR (G.) (1967). - c) III. Mitt. : Die Verteilung der Typen des Sc. agalactiae in den Rinderbeständen des Bezirkes Gera. Arch. Exp. Vet. Med., 21, 65-76.

24. Plummer (H.) (1935). - A serological study of hemolytic Streptococci. J. Bact., 30, 5-20.

25. Pomales-Lebron (A.), Morales-Otero (P.) and Baralt (J.) (1947). - Biological properties and mouse virulence of Streptococcus agalactiae and Lancefields group G-Streptococci from human sources. Proc. Soc. Exp. Biol. Med., 64, $410-412$.

26. SCHRöTER (G.) (1968). - Kleinraumepidemiologische Untersuchungen zur Neugeborenenmengitis. Arch, Hyg. u, Bakt., 152, 488.

27. Schröter (G.) (1968). - Das Vorkommen von B-Galaktosidase bei Streptokokken der serologischen Gruppe B. Zbl. Bart. I. Abt. Orig. 1968, 206, 45-51.

28. Seelemann (M.) (1963). - Zur Frage der Pathogenität des Streptococcus agalactiae (Galtstreptococcus) und der Gesundheitsschädlichkeit der Milch von mastitiskranken Kühen für den Menschen. Mh. Tierheilk., 15, 199-210.

29. Shimzu (K.) u. a. (1959). - Verbreitung von Streptococcus agalactiae bei den Haustieren und langfristige Beobachtungen eines Euters, das den Keim beherbergte. Jap. J. vet. Res., 7, 203-214.

30. SPASIC (J. M.) (1966). - Bedeutung einiger biologischer Charakteristiken der Gruppe B-Streptokokken für die Entstehung und Verbreitung der Mastitis bei den Kühen und der Infektion bei den Menschen. Acta Vet. Beogr. 1966 XVI, 171.

31. Turner (E.) (1965). - Neonatal menigitis. Pathol. Biol., 10, 985.

32. Wahl (R.), Cayeux (P.) et Derlot (E.) (1962). - Méningite du nouveau-né et du nourrisson à streptocoque du groupe B. Path. Biol., 10, 985.

33. Winterbauer (R. H.) (1966). - Unusual occurence of neonatal menigitis due to group B Beta-Hemolytic Streptococci. Pediatrics, Vol. 38, No 4, Part I.

Adresse des auteurs : Dr G. Hahn, Dr W. Heeschen, Dr J. Reichmuth, Prof. Dr A. Tolle, Institut für Hygiene der Bundesanstalt für Milchforschung, 2300 Kiel, Hermann-Weigmann-Str. 1-27. 